\title{
BLOWUP OF SOLUTIONS AND BOUNDARY INSTABILITIES IN NONLINEAR HYPERBOLIC EQUATIONS*
}

\author{
ROBIN YOUNG ${ }^{\dagger}$
}

\begin{abstract}
We construct elementary examples of systems of hyperbolic equations having solutions which blow up in finite time. We explicitly describe the system, initial data and solution. First, we exhibit a $3 \times 3$ system with compactly supported data which blows up in finite time. The solution blows up in amplitude ( $L^{\infty}$ norm) on an entire interval, so there is no possibility of continuing the solution beyond the blowup time. We then consider a system of two Burgers equations which are coupled through linear boundary conditions. We record the interesting observation that although the IBVP with a single boundary condition is globally well-posed, when two boundary conditions are used on a finite domain, the IBVP is ill-posed. Because waves are reflected back into the domain, multiple interactions combine to give blowup in finite time, for arbitrarily small initial data. We conclude that some global integral or energy condition must be imposed in order to expect stability of solutions to IBVPs on compact domains. Finally, we show that the presence of shocks is not necessary, by exhibiting solutions which are continuous in the nonlinear fields. However, our solutions do contain discontinuities in the linearly degenerate field.
\end{abstract}

\section{Introduction}

Glimm's celebrated existence theorem for hyperbolic conservation laws states that weak solutions can be globally defined, provided that the initial data be small enough in the total variation norm [3]. This was made more precise in [14, 13], in which the author showed that global solutions exist provided that the total variation of the data lie below a critical threshold, and the sup-norm be small enough. For general systems, a small sup-norm is needed to solve Riemann problems [9], while the Glimm functional for the total variation bounds the effects of all future nonlinear wave interactions. The threshold arises because nonlinear interaction effects can be measured by a geometric series, which must sum to provide global bounds. This threshold is not present for $2 \times 2$ systems, for which the Glimm-Lax decay theory applies.

It is well known that in general, global solutions may not exist if the total variation of the data is beyond the threshold. This is essentially due to a Ricatti-type blowup in wave amplitudes due to multiple interaction effects. Nonlinear instability was first demonstrated for $3 \times 3$ hyperbolic systems by Hunter [5], using an asymptotic expansion which was made rigorous by Joly, Metivier and Rauch [8]. In [15], the author found several explicit examples of solutions to $3 \times 3$ systems which exhibit nonlinear instability, and which clearly show the cause of instability to be the cumulative effect of many interactions of small waves. Some of these systems were then modified by Jenssen, who found solutions which actually blow up to infinity in finite time [6]. This is accomplished by having many interactions occur inside a wedge formed by two approaching shocks, so that there is an infinite number of (unstable) interactions in finite time. This same mechanism has been shown to work in systems of three genuinely nonlinear families [1].

Following his own work on regularity of solutions to Hamilton-Jacobi equations [11], Bo Su posed the question of whether, when the system is genuinely nonlinear and the wavespeed grows rapidly, blowup of the solution can be avoided. Here we

\footnotetext{
* Received: July 18, 2002; accepted (in revised version): November 4, 2002.

$\dagger$ I thank Bo Su for bringing this question to my attention. This work was partially supported by the National Science Foundation under grant number DMS-0104485.

‡ Department of Mathematics \& Statistics University of Massachusetts, Amherst, MA 01003, USA.
} 
show that this is not the case, and indeed growth in wavespeed causes interactions to occur more frequently, in turn leading to growth in amplitude. As this process continues, both states and wavespeeds approach infinity in finite time. Moreover, the solution tends to infinity on the entire support of the initial data, so there is no way of continuing the solution beyond the blowup time.

As in [15], we construct explicit piecewise constant solutions which have prescribed interactions. Our construction is as follows: assume a $3 \times 3$ system with forward, stationary and backward waves; starting with two stationary waves, start with a (forward or backward) shock between them. We choose our system so that the interaction of the shock with a standing wave has the effect of reflecting a stronger shock. This in turn reflects another shock, and the pattern repeats. Since stronger shocks travel faster, the time between interactions decreases, and by summing another geometric series, we get infinitely many interactions and blowup in finite time.

Our $3 \times 3$ system can be regarded as an interpolation of $2 \times 2$ systems, with a Riemann coordinate which changes across the standing waves acting as interpolation parameter. The system has the form

$$
\begin{aligned}
u_{t}+f(u, S, w)_{x} & =0, \\
S_{t} & =0, \\
w_{t}-g(u, S, w)_{x} & =0,
\end{aligned}
$$

where $f$ and $g$ are chosen in such a way that the different $2 \times 2$ fluxes are given by setting $S=0$ and $S=1$, respectively. This means that we can treat several different fluxes and we have a number of tunable parameters. In a future paper [12], we will carry out a bifurcation analysis which describes the transition from stability, when Glimm's theorem applies, to instability, when blowup occurs.

It is well known that the existence of a uniformly convex entropy implies that the $L^{2}$ norm of the solution is non-increasing, ruling out the blowup described here. Thus, our interpolated systems do not have a convex entropy, although the existence of a nonconvex entropy has not been ruled out. Regarding the $3 \times 3$ flux as a homotopy of $2 \times 2$ fluxes, we would like to construct a nonconvex entropy as a homotopy of $2 \times 2$ entropies. Our results do indicate that general perturbations of fluxes must be handled with great care; any general $O(1)$ perturbation of flux and/or data may lead to strong instabilities. It remains an open question whether a convex entropy is sufficient to rule out strong nonlinear instabilities.

We note that unlike the systems in [15], the genuine nonlinearity of the interior system is an essential ingredient in this construction. The increasing speed of reflected waves ensures that the time between interactions decreases, and that we actually get infinitely many interactions in finite time. If the wavespeeds were linear, the time between interactions would be constant, and we could not get finite time blowup. Although genuine nonlinearity is essential, the formation and presence of shock waves is not an essential ingredient for blowup. Indeed, in the final section, we examine solutions that consist of compression waves and again blow up in finite time, before the compressive waves break down and shock waves are formed.

Returning to $2 \times 2$ systems, it is clear that we can generate the same unstable wave pattern if we allow the $2 \times 2$ flux to be spatially dependent and discontinuously varying. A much more interesting observation is that the same interaction effect can be modeled by boundary conditions. Thus, we get blowup of solutions of an initial boundary value problem (IBVP) for a $2 \times 2$ nonlinear system. 
Our $2 \times 2$ system is particularly simple: it can be taken to be a pair of uncoupled Burgers' equations,

$$
\begin{aligned}
u_{t}+\left(\frac{1}{2} u^{2}\right)_{x} & =0, \\
w_{t}-\left(\frac{1}{2} w^{2}\right)_{x} & =0,
\end{aligned}
$$

on the domain $\{(x, t) \mid 0<x<1, t>0\}$, together with linear boundary conditions

$$
\begin{aligned}
& u-\beta w=0 \quad \text { at } \quad x=0, \quad \text { and } \\
& w-\beta u=0 \quad \text { at } \quad x=1 \text {. }
\end{aligned}
$$

For this IBVP, we show that there are weak solutions with arbitrarily small data which blow up in finite time, and which take on the boundary data in the strong sense. In contrast, we show that the corresponding one-sided IBVP on an open domain $x>0$, with the same boundary condition at $x=0$, is globally well-posed independent of the size of the initial data. Again, the cause of instability is multiple interactions with the boundary, while a single interaction is not enough to generate instability. System (1.2), being a pair of uncoupled scalar equations, clearly has many convex entropies.

We draw the important conclusion that in order to have stability of solutions of IBVPs on compact domains, we require more than the usual local conditions of counting characteristics at a boundary. Indeed, it appears that we need a global (integral) condition expressing the fact that the total energy of the system be conserved. Such a condition would provide an essential physically meaningful coupling of the boundary conditions with the interior system of equations.

The paper is arranged as follows: in the next section, we construct the flux and describe those individual waves in our construction. We then consider interactions and show that each reflected shock is stronger and faster than the incident shock. We next carry out the inductive proof that the solution blows up, and does so in finite time. In the following section, we show that the blowup can be generated by twosided linear boundary conditions, while the one-sided IBVP is well-posed. Finally, we show that the blowup is independent of the gradient blowup characteristic of shock formation, by replacing the shocks in the earlier sections by compressive waves. Note that although there are no shocks in this solution, the solution is discontinuous along the vertical lines $x= \pm \ell$. Whether or not blowup is possible for classical solutions with no discontinuities remains an open question.

\section{The Construction}

Because we are working with piecewise constant solutions, our analysis is essentially algebraic. We start with two stationary waves, say at $x=0$ and $x=1$. We then place a forward shock at the origin. This interacts with the standing wave at $x=1$, and a backward wave is reflected. In order to preserve piecewise constancy of the solution, we want this reflected wave to be a shock. We want this reflected shock to be stronger, which in turn implies it has a greater speed. By symmetry, if we can accomplish this for one general interaction, the pattern repeats and we can inductively construct a piecewise constant solution. Once we know the shock speeds, we calculate the time between successive interactions of forward shocks with the right stationary wave. If the shock speed increases by a uniform factor at each interaction, then the time between interactions decreases by that uniform factor, and we sum a geometric series to get an infinite number of interactions in finite time. We thus wish 
to construct the wave pattern of Figure 2.1, and exactly describe the states and times of interaction therein.

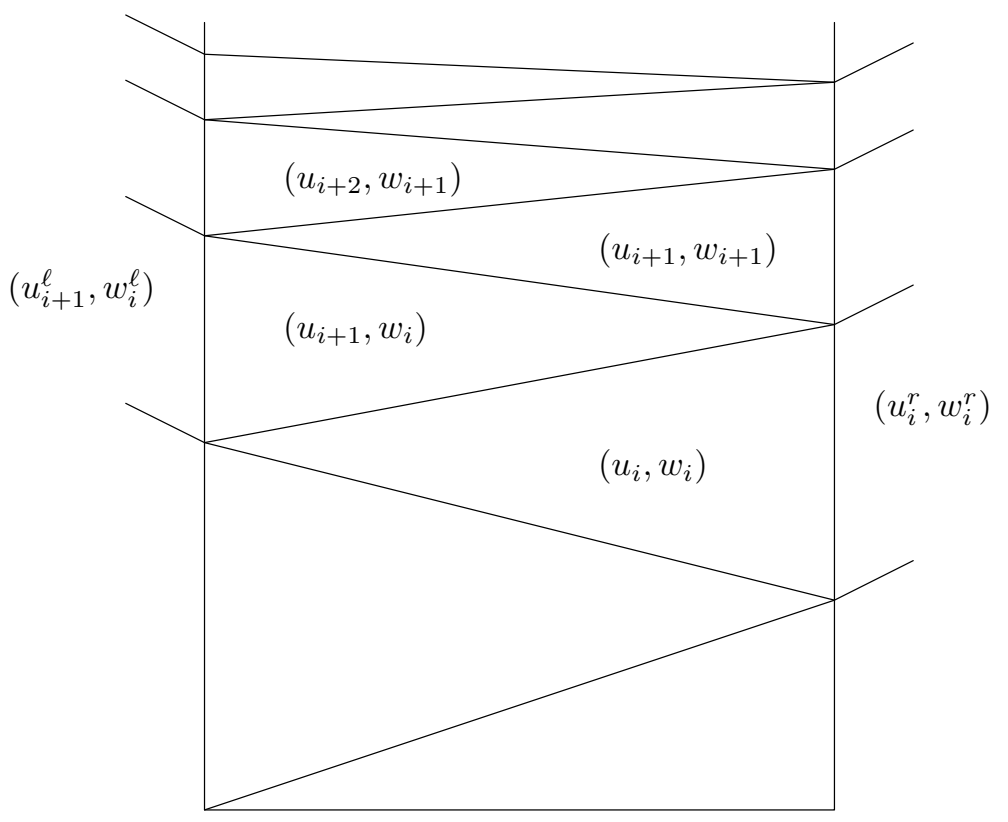

FIG. 2.1. The wave pattern

2.1. The Flux. We describe a general state by its three components $u, S$ and $w$. Generally $u$ will be a coordinate for forward waves, $w$ for backward waves, and $S$ will be the Riemann coordinate which describes stationary waves. Since we want $S$ to have zero wavespeed, we simply take the equation for $S$ to be

$$
S_{t}=0
$$

Note that this choice of equation for $S$ allows us to effectively consider $2 \times 2$ systems with spatially varying coefficients. Indeed, our results apply unchanged for such $2 \times 2$ systems, provided the coefficients are allowed to change discontinuously. Referring to the figure, we take this component of our solution to be simply

$$
S(x, t)=S_{0}(x)=1 \quad \text { for } \quad 0<x<1,
$$

and zero otherwise.

We now choose two different $2 \times 2$ fluxes, one inside the strip $0<x<1$, and one outside. For simplicity, inside the strip we use two uncoupled Burgers' fluxes for the forward and backward waves,

$$
\begin{aligned}
u_{t}+\left(u^{2} / 2\right)_{x} & =0 \quad \text { and } \\
w_{t}-\left(w^{2} / 2\right)_{x} & =0
\end{aligned}
$$

for $S \equiv 1$. Since these are uncoupled, only $u$ changes across a forward wave, while only $w$ changes across a backward wave inside the strip $0<x<1$. 
Outside the strip $0<x<1$, we again choose a convenient $2 \times 2$ flux. It is easiest and most convenient to simply take this $2 \times 2$ flux to be linear in $u$ and $w$. This choice ensures that there are no wave interactions outside the strip, so our solution will be completely determined everywhere on its domain, and also simplifies interactions with standing waves. Outside the strip, for $S \equiv 0$, we thus write

$$
\left(\begin{array}{c}
u \\
w
\end{array}\right)_{t}+A\left(\begin{array}{c}
u \\
w
\end{array}\right)_{x}=0
$$

where we will choose the matrix $A$ so that the wave interactions reflect shocks which are stronger than the incident shocks. This matrix will be chosen by specifying its eigensystem, or equivalently the behavior of the (linear) waves. It is simplest to take the (constant) forward and backward wavespeeds to be $c$ and $-c$, respectively. Without loss of generality, we take $A$ to have the form

$$
A=\left(\begin{array}{cc}
a & b \\
-b & -a
\end{array}\right), \quad \text { where } \quad c^{2}=a^{2}-b^{2}>0,
$$

and $a$ and $b$ will be chosen when we consider interactions. Note that only forward waves leave the strip at the right edge, and only backward waves leave the left edge. Moreover, since the flux is linear outside of the strip, there are no interactions there.

We now give the conservative flux as a function of all three variables $u, S$ and $w$ : since our construction has only treated the discrete cases $S=0$ and $S=1$, and each is in conservation form, we simply interpolate between the two fluxes. Using a linear interpolation, we take the $2 \times 2$ flux for $u$ and $w$ to be the vector

$$
\left(\begin{array}{c}
f \\
g
\end{array}\right)=S\left(\begin{array}{c}
u^{2} / 2 \\
-w^{2} / 2
\end{array}\right)+(1-S) A\left(\begin{array}{c}
u \\
w
\end{array}\right),
$$

so that with $A$ as in (2.5), the full $3 \times 3$ system has the form

$$
\begin{aligned}
u_{t}+\left(S u^{2} / 2+(1-S) a u+(1-S) b w\right)_{x} & =0, \\
S_{t} & =0, \\
w_{t}-\left(S w^{2} / 2+(1-S) b u+(1-S) a w\right)_{x} & =0 .
\end{aligned}
$$

Equivalently, we write

$$
\begin{aligned}
u_{t}+f(u, S, w)_{x} & =0, \\
S_{t} & =0, \\
w_{t}-f(w, S, u)_{x} & =0,
\end{aligned}
$$

where $f$ is given by

$$
f(p, S, q)=S p^{2} / 2+(1-S) a p+(1-S) b q,
$$

and $a$ and $b$ are constants to be chosen.

2.2. The Waves. We now describe those waves that occur for our system (2.8), for the reduced cases of $S=0$ and $S=1$. Since our solutions are piecewise constant, we consider only shock waves and contact discontinuities. In a later paper [12], we will describe all nonlinear waves and all ranges of $S$. 
The shocks and jump discontinuities are described by the usual Rankine-Hugoniot conditions,

$$
\begin{aligned}
\sigma[u] & =[f(u, S, w)], \\
\sigma[S] & =0, \\
\sigma[w] & =[-f(w, S, u)],
\end{aligned}
$$

where $\sigma$ is the speed of the discontinuity and [.] represents the jump in a quantity. It is clear that either $S$ is constant across the wave (either 0 or 1 in our case) or $\sigma=0$. In our construction, $\sigma=0$ corresponds to the edges of the strip, $x=0$ or $x=1$.

If $\sigma \neq 0$, then $S$ is constant across the shock, and we are simply dealing with a 2-D flux. We are considering only $S=0$ and $S=1$, for which the corresponding fluxes are linear and uncoupled Burgers', and the equations reduce to (2.4) and (2.3), respectively.

Inside the strip, taking $S=1,(2.10)$ reduces to

$$
\sigma[u]=\left[\frac{1}{2} u^{2}\right] \quad \text { and } \quad \sigma[w]=\left[-\frac{1}{2} w^{2}\right]
$$

so that either

$$
\begin{aligned}
& \sigma=\bar{u}=\frac{u_{+}+u_{-}}{2} \quad \text { and } \quad[w]=0, \quad \text { or } \\
& \sigma=-\bar{w}=-\frac{w_{+}+w_{-}}{2} \quad \text { and } \quad[u]=0,
\end{aligned}
$$

where the subscripts denote the states on either side of the discontinuity. In particular, for $S=1$, only one of the variables changes across the wave. Taking both $u$ and $w$ to be positive inside the strip, these are forward and backward waves, respectively. The entropy conditions for these waves require that the characteristic speeds (respectively $u$ and $-w$ ) impinge on the shock from both sides. This implies that the state $u$ or $w$ should increase behind the shock, or towards later times. Thus, in our construction, the requirement that the waves inside the strip be shocks of increasing speed is the same as the requirement that $u$ and $w$ should increase in time.

Outside the strip $0<x<1$, we have $S=0$ and (2.10) reduces to

$$
\sigma\left(\begin{array}{c}
{[u]} \\
{[w]}
\end{array}\right)=A\left(\begin{array}{c}
{[u]} \\
{[w]}
\end{array}\right)
$$

so the eigenvalues of $A$ give the wavespeeds and the eigenvectors describe the changes of state across the waves. According to (2.5), the eigenvalues are $\pm c$, and if the corresponding left eigenvectors are $\ell_{ \pm}$, then the forward waves in the region $x>1$ satisfy

$$
\ell_{-} \cdot\left(\begin{array}{c}
{[u]} \\
{[w]}
\end{array}\right)=0 \quad \text { with speed } \quad c>0
$$

and similarly the backward waves in the region $x<0$ satisfy

$$
\ell_{+} \cdot\left(\begin{array}{c}
{[u]} \\
{[w]}
\end{array}\right)=0 \quad \text { with speed } \quad-c<0 .
$$

Our choice of the left eigenvectors $\ell_{ \pm}$will prove to be critical in describing the wave interactions and generating blowup. 
We now describe the stationary jumps, for which $[S] \neq 0$ but $\sigma=0$. In this case the jump relations $(2.10)$ reduce to

$$
[f(u, S, w)]=0 \quad \text { and } \quad[f(w, S, u)]=0 .
$$

Since we are interested only in jumps between $S=0$ and $S=1$, we get the simple form

$$
\left(\begin{array}{c}
\frac{1}{2} u_{1}^{2} \\
-\frac{1}{2} w_{1}^{2}
\end{array}\right)=A\left(\begin{array}{c}
u_{0} \\
w_{0}
\end{array}\right)
$$

where the subscripts denote the corresponding value of $S$. Here we again see that $S$ indeed acts as a switch between the Burgers' and linear fluxes.

We now have a complete description of the waves appearing in our construction, which can readily be extended to all nonlinear waves to yield a general solution to the Riemann problem, (see [12]).

2.3. Interactions. We now consider the interaction appearing in our construction, namely a shock inside the strip $0<x<1$ reaching the boundary and transmitting a linear wave. Suppose the interaction occurs at the right edge $x=1$, so that the incoming shock is a forward shock, and the wave reflected back into the strip will be a backward shock. We denote the states inside the strip (in increasing time) by

$$
\left(u_{a}, w_{a}\right), \quad\left(u_{b}, w_{a}\right) \text { and }\left(u_{b}, w_{b}\right)
$$

Here we have used the fact that $w$ (resp. $u$ ) is unchanged across a forward (resp. backward) shock, and the subscripts $a$ and $b$ stand for the states ahead of and behind the appropriate shock, respectively.

Our problem is the following: given the incoming states $u_{a}<u_{b}$ and $w_{a}$, to choose the eigenvector $\ell_{-}$of $A$ in such a way that the reflected wave is a shock, so $w_{b}>w_{a}$, and such that it has a greater absolute speed than the incoming shock,

$$
\frac{w_{b}+w_{a}}{2}>\frac{u_{b}+u_{a}}{2} .
$$

Note that by choosing the eigenvectors $\ell_{ \pm}$and linear speed $c$, we fully determine the matrix $A$, and the states outside the strip are determined by (2.16). Thus, we do not explicitly choose data outside the strip.

According to (2.16), the state outside the strip and ahead of the linear wave, say $\left(u_{a}^{0}, w_{a}^{0}\right)$, satisfies

$$
\left(\begin{array}{c}
\frac{1}{2} u_{a}^{2} \\
-\frac{1}{2} w_{a}^{2}
\end{array}\right)=A\left(\begin{array}{c}
u_{a}^{0} \\
w_{a}^{0}
\end{array}\right)
$$

while the state behind the linear wave and outside the strip, say $\left(u_{b}^{0}, w_{b}^{0}\right)$, satisfies

$$
\left(\begin{array}{c}
\frac{1}{2} u_{b}^{2} \\
-\frac{1}{2} w_{b}^{2}
\end{array}\right)=A\left(\begin{array}{c}
u_{b}^{0} \\
w_{b}^{0}
\end{array}\right) \text {. }
$$

We now relate the states outside the strip by (2.14): since they are joined by a forward jump, we have

$$
\ell_{-} \cdot\left(\begin{array}{c}
u_{b}^{0} \\
w_{b}^{0}
\end{array}\right)=\ell_{-} \cdot\left(\begin{array}{c}
u_{a}^{0} \\
w_{a}^{0}
\end{array}\right)
$$


Combining this with (2.19) and (2.20), we describe the interaction as

$$
\ell_{-} \cdot\left(\begin{array}{c}
\frac{1}{2} u_{b}^{2} \\
-\frac{1}{2} w_{b}^{2}
\end{array}\right)=\ell_{-} \cdot\left(\begin{array}{c}
\frac{1}{2} u_{a}^{2} \\
-\frac{1}{2} w_{a}^{2}
\end{array}\right),
$$

and we regard this as giving $w_{b}$ as a function of $u_{a}, u_{b}$ and $w_{a}$.

The interaction at the left edge of the strip is entirely symmetric: we take the incident states to be $\left(u_{a}, w_{a}\right)$ and $\left(u_{a}, w_{b}\right)$ and solve for the outgoing state $\left(u_{b}, w_{b}\right)$. Describing the waves in the same way, we get the identity

$$
\ell_{+} \cdot\left(\begin{array}{c}
\frac{1}{2} u_{b}^{2} \\
-\frac{1}{2} w_{b}^{2}
\end{array}\right)=\ell_{+} \cdot\left(\begin{array}{c}
\frac{1}{2} u_{a}^{2} \\
-\frac{1}{2} w_{a}^{2}
\end{array}\right)
$$

giving $u_{b}$ as a function of $w_{a}, w_{b}$ and $u_{a}$.

We now choose the left eigenvectors of the matrix $A$ to ensure that our individual interactions are unstable. If we choose

$$
\ell_{-}=(\beta, 1) \quad \text { and } \quad \ell_{+}=(1, \beta),
$$

then (2.21) becomes

$$
w_{b}^{2}-w_{a}^{2}=\beta\left(u_{b}^{2}-u_{a}^{2}\right),
$$

while (2.22) becomes

$$
u_{b}^{2}-u_{a}^{2}=\beta\left(w_{b}^{2}-w_{a}^{2}\right) .
$$

In both cases the interaction will produce a stronger reflected shock provided we take $\beta>1$.

We remark that although there appears to be a choice of sign in (2.24) and (2.25), we must take the sign of $w_{b}$ to be that of $w_{a}$ in (2.24) in order to satisfy the entropy condition. Since $\left|w_{b}\right|>\left|w_{a}\right|$, if we chose the negative root, the reflected wave would be a rarefaction from $w_{a}>0$ to $w_{b}<0$, and the right half of the rarefaction, with speed $-w>0$, would not be confined to the strip.

2.4. The Induction. We are now in a position to describe the wave pattern of Fig. 2.1 fully. Referring to the figure, we label the states inside the strip as $\left(u_{i}, w_{i}\right)$ and $\left(u_{i+1}, w_{i}\right)$, where these two states are spanned by a forward shock, and the states $\left(u_{i+1}, w_{i}\right)$ and $\left(u_{i+1}, w_{i+1}\right)$ are spanned by a backward shock. We now use our descriptions of a single interaction to get a difference equation relating successive values of $u_{i}$ and $w_{i}$.

According to (2.24), the interactions at the right edge of the strip $x=1$ give

$$
w_{i+1}^{2}-w_{i}^{2}=\beta\left(u_{i+1}^{2}-u_{i}^{2}\right),
$$

while from (2.25), those at the left edge $x=0$ give

$$
u_{i+2}^{2}-u_{i+1}^{2}=\beta\left(w_{i+1}^{2}-w_{i}^{2}\right) .
$$

Combining these, we see that both $z_{i}=u_{i}^{2}$ and $z_{i}=w_{i}^{2}$ satisfy the linear difference equation

$$
z_{i+2}-z_{i+1}=\beta^{2}\left(z_{i+1}-z_{i}\right)
$$


This difference equation has general solution

$$
z_{n}=c_{0}+d_{0} \beta^{2 n}
$$

where the constants $c_{0}$ and $d_{0}$ are determined from the initial values $z_{0}$ and $z_{1}$.

In our case, choice of initial conditions corresponds to choice of the initial shock, given in terms of $u_{0}$ and $u_{1}$, and of the initial value $w_{0}$. The second initial condition for $w$ is then given by (2.26) with $i=0$. Taking all values of $u$ and $w$ to be positive inside the strip, we see that the initial forward wave will be a shock if $u_{1}>u_{0}$, in which case we have

$$
\begin{aligned}
& d_{0}=\frac{z_{1}-z_{0}}{\beta^{2}-1}=\frac{u_{1}^{2}-u_{0}^{2}}{\beta^{2}-1}>0 \text { and } \\
& c_{0}=\frac{\beta^{2} z_{0}-z_{1}}{\beta^{2}-1}=\frac{\beta^{2} u_{0}^{2}-u_{1}^{2}}{\beta^{2}-1} .
\end{aligned}
$$

In particular, if $u_{1}=\beta u_{0}$, then our solution simplifies to

$$
u_{n}=\beta^{n} u_{0},
$$

and since $u_{0}>0$ and $\beta>1, u_{n} \rightarrow \infty$ as $n \rightarrow \infty$.

With these choices of $u_{0}$ and $u_{1}$, it is also convenient to take $w_{1}=\beta w_{0}$, which by (2.26) reduces to the assumption $w_{0}=\sqrt{\beta} u_{0}$, and for which we get the solution

$$
w_{n}=\beta^{n} w_{0}=\beta^{n+\frac{1}{2}} u_{0}
$$

which is also unbounded as $n \rightarrow \infty$. Note that these assumptions are only conveniences: the only condition that is necessary for our construction is that the initial wave and first reflected wave be shocks, which will be the case if $u_{1}>u_{0}$, which in turn implies also $w_{1}>w_{0}$.

Our final step is to show that an infinite number of interactions occur in finite time, so $n \rightarrow \infty$ and the solution blows up in both $u$ and $w$ in finite time. Since inside the strip we are simply dealing with Burgers equations, by (2.12) we know that the shock speeds are given by

$$
\sigma_{i}^{+}=\frac{\left(u_{i}+u_{i+1}\right)}{2} \text { and } \sigma_{i}^{-}=-\frac{\left(w_{i}+w_{i+1}\right)}{2}
$$

respectively. Since the ends of the strip are unit distance apart, the time between successive interactions is thus

$$
\Delta t_{i}^{+}=\frac{2}{\left(u_{i}+u_{i+1}\right)}, \quad \text { and } \quad \Delta t_{i}^{-}=\frac{2}{\left(w_{i}+w_{i+1}\right)},
$$

respectively, the superscripts corresponding to the direction of the wave.

We now sum these times between successive interactions to find the time at which the $2 J$-th interaction occurs,

$$
t_{2 J}=\sum_{j \leq 2 J} \Delta t_{j}^{ \pm}=\sum_{j \leq J} \frac{2}{\left(u_{j}+u_{j+1}\right)}+\sum_{j \leq J} \frac{2}{\left(w_{j}+w_{j+1}\right)} .
$$

Using (2.31) and (2.32), we see that this is a pair of geometric series, both of which converge. Indeed, we get

$$
\begin{aligned}
t_{2 J} & =\frac{2}{u_{0}+u_{1}} \sum_{j \leq J} \beta^{-j}+\frac{2}{w_{0}+w_{1}} \sum_{j \leq J} \beta^{-j} \\
& \leq \frac{2}{1+\beta}\left(\frac{1}{u_{0}}+\frac{1}{w_{0}}\right) \frac{1}{\beta-1}=t_{*},
\end{aligned}
$$


for each $J$. This clearly determines a critical time $t_{*}$ before which infinitely many interactions happen, and the amplitude of the solution blows up to infinity.

We finally observe that by choosing $w_{0}=\sqrt{\beta} u_{0}$ inside the strip, and zero initial data outside the strip, we get the pattern we have described with two outgoing linear waves which leave the strip at time 0 . To see this, we solve the initial Riemann problems. At the left corner of the strip, we solve the Riemann problem with zero on the left and $\left(u_{0}, 1, w_{0}\right)$ on the right. The solution consists of a backward linear wave, stationary jump, and forward nonlinear wave for which the behind state is $\left(u_{1}, 1, w_{0}\right)$. Using (2.15) and (2.16), we get the relation

$$
\ell_{+} \cdot\left(\begin{array}{c}
\frac{1}{2} u_{1}^{2} \\
\frac{1}{2} w_{0}^{2}
\end{array}\right)=0
$$

and by (2.23), this yields

$$
u_{1}=\sqrt{\beta} w_{0}=\beta u_{0},
$$

consistent with our initial choice. Similarly, at the right corner of the strip, we get a forward linear wave, stationary jump, and backward nonlinear wave, behind which we have $\left(u_{0}, 1, \tilde{w}_{0}\right)$. Using $(2.14),(2.16)$ and $(2.23)$, we derive

$$
\ell_{-} \cdot\left(\begin{array}{c}
\frac{1}{2} u_{0}^{2} \\
\frac{1}{2} \tilde{w}_{0}^{2}
\end{array}\right)=0
$$

which yields

$$
\tilde{w}_{0}=\sqrt{\beta} u_{0}=w_{0},
$$

and so there is no backward nonlinear wave from this corner.

We note that our choice of initial state $\left(u_{0}, w_{0}\right)$ is not the only one which gives a solution which blows up. Since the equations for $u$ and $w$ are uncoupled in the strip $0<x<1$, any state $\left(u_{0}, w_{0}\right)$ which yields two shocks into the strip will lead to blowup, because forward and backward waves do not interact. Equations (2.37) and (2.39) still hold, and both waves will be shocks provided both

$$
\begin{aligned}
& u_{1}=\sqrt{\beta} w_{0} \geq u_{0} \text { and } \\
& \tilde{w}_{0}=\sqrt{\beta} u_{0} \geq w_{0},
\end{aligned}
$$

which holds provided

$$
0<u_{0} \leq \sqrt{\beta} w_{0} \leq \beta u_{0} .
$$

We also expect blowup for more general initial data, provided some condition analogous to (2.42) holds. More general initial data will be considered elsewhere [12].

Our construction is based on the assumption $\beta>1$, which we now relate directly to the matrix $A$ defined by (2.5). The eigenvalues of $A$ are $\pm c$, where $c=\sqrt{a^{2}-b^{2}}$. The corresponding left eigenvectors satisfy

$$
\ell_{-} \cdot\left(\begin{array}{cc}
a+c & b \\
-b & -a+c
\end{array}\right)=0 \text { and } \ell_{+} \cdot\left(\begin{array}{cc}
a-c & b \\
-b & -a-c
\end{array}\right)=0,
$$

and so have the form (2.23) for $\beta$ given by

$$
\beta=\frac{b}{a+c}=\frac{a-c}{b} .
$$


It is routine to check that $\beta>1$ only if both $b<0$ and $a<0$, and so our condition on the entries of the matrix $A$ becomes simply

$$
a<b<0 .
$$

We have proven the following theorem.

THEOREM 1. For the $3 \times 3$ system of conservation laws (2.8), (2.9) with (2.45), there are solutions which blow up in finite time $t_{*}$ depending on the data. Moreover, the states become infinite at time $t_{*}$ on the entire support of the initial data, and there is no way to continue the solution beyond time $t_{*}$. The data consists of the single constant state $\left(u_{0}, 1, w_{0}\right)$, supported on the interval $0 \leq x \leq 1$, and where $u_{0}$ and $w_{0}$ can be made arbitrarily small, provided (2.42) holds.

We note that this does not violate Glimm's existence theorem because the total variation of the initial data is not small. Glimm's estimate rests on the ability to sum a geometric series for the total variation, which in this case will diverge. In a future paper [12], we will show that instability holds if $S_{0} \neq 1$, and we will examine the transition from stability (with $S_{0} \approx 0$ ), to instability (with $S \approx 1$ ).

The solution outside the strip consists of forward and backward linear waves, each emanating from a point of interaction. We can describe the resulting states and jumps using (2.16): if we write $\left(u_{j}^{r}, w_{j}^{r}\right)$ for the state to the right of $\left(u_{j}, w_{j}\right)$ as in Fig. 2.1, then (2.16) gives

$$
\left(\begin{array}{c}
u_{j}^{r} \\
w_{j}^{r}
\end{array}\right)=A^{-1}\left(\begin{array}{c}
\frac{1}{2} u_{j}^{2} \\
-\frac{1}{2} w_{j}^{2}
\end{array}\right)
$$

and by (2.31) and (2.32), this can be written

$$
\begin{aligned}
\left(\begin{array}{c}
u_{j}^{r} \\
w_{j}^{r}
\end{array}\right) & =\frac{\beta^{2 j}}{2} A^{-1}\left(\begin{array}{c}
u_{0}^{2} \\
-\beta u_{0}^{2}
\end{array}\right) \\
& =\frac{\beta^{2 j} u_{0}^{2}}{2\left(a^{2}-b^{2}\right)}\left(\begin{array}{c}
a-b \beta \\
a \beta-b
\end{array}\right) .
\end{aligned}
$$

Now, according to (2.44), we have $a-b \beta=c$ and $a \beta-b=-\beta c$, so (2.47) becomes

$$
\begin{aligned}
& u_{j}^{r}=\frac{\beta^{2 j} u_{0}^{2}}{2 c}=\frac{u_{j}^{2}}{2 c}>0 \text { and } \\
& w_{j}^{r}=-\frac{\beta^{2 j+1} u_{0}^{2}}{2 c}=-\frac{w_{j}^{2}}{2 c}<0 .
\end{aligned}
$$

This is unsurprising because by taking the initial external state to be zero, we have that each of the vectors $\left(u_{j}^{r}, w_{j}^{r}\right)^{T}$ is a right eigenvector of $A$, and (2.48) again follows from (2.46). Similarly, the states to the left of the strip are

$$
u_{j+1}^{\ell}=-\frac{u_{j+1}^{2}}{2 c}<0 \quad \text { and } \quad w_{j}^{\ell}=\frac{w_{j}^{2}}{2 c}>0 .
$$

We now see what the solution $u$ looks like for finite times: on the left of the strip, $u$ is piecewise constant decreasing from 0 to $u_{j+1}^{\ell} ; u$ jumps from $u_{j+1}^{\ell}$ to $u_{j+1}$ at $x=0$; in the strip $u$ may jump to $u_{j}$; then at the right edge, $u$ jumps to $u_{j}^{r}$, and to the right of the strip $u$ is monotone decreasing back to 0 . In particular, although $u$ takes on some negative values, as it must for the integral $\int u$ to be conserved; there 


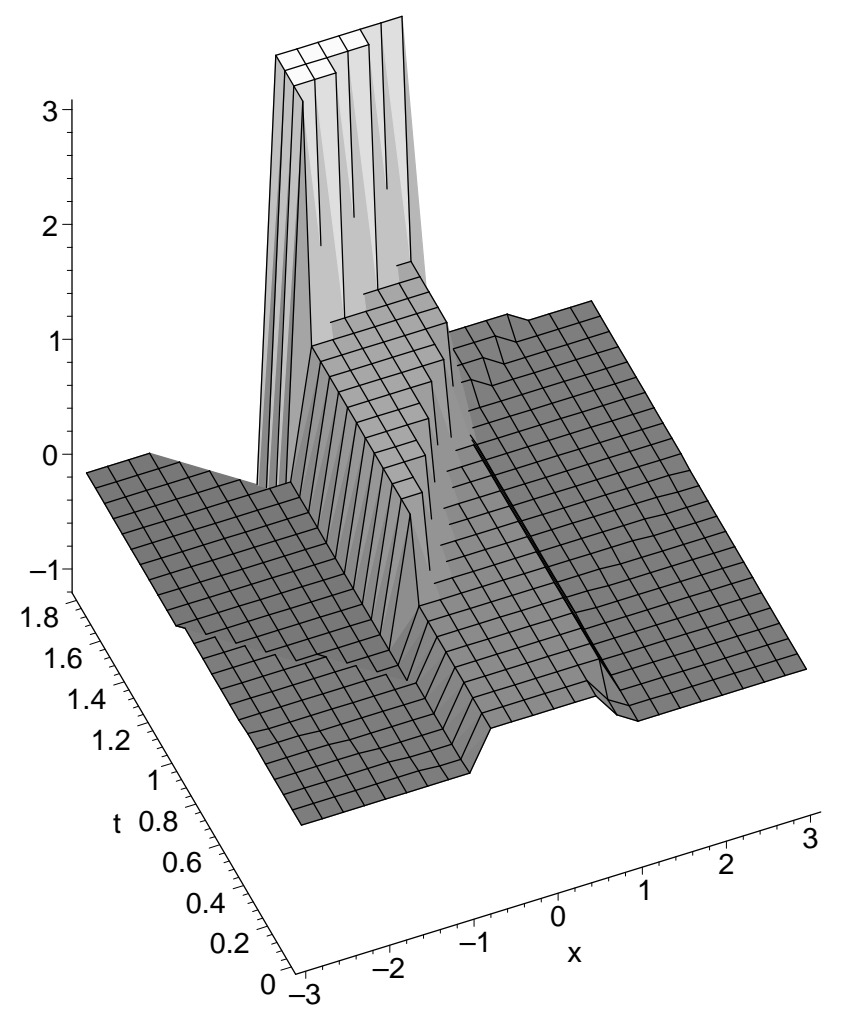

FIG. 2.2. Graph of the solution $u(x, t)$

are no wild oscillations. Note that negative values of $u$ are created only at the left edge, corresponding to the interactions at which a stronger shock is reflected back into the strip. Since $\int u$ must be conserved and a more positive value of $u$ is reflected, the state behind the transmitted wave must necessarily have a larger negative value.* This does not occur (for $u$ ) for the waves transmitted at the right edge of the strip. See 2.2 for a plot of the solution $u=u(x, t)$ which blows up. Entirely similar remarks hold for the other component $w$, for which we have

$$
w(x, t)=u(1-x, t) .
$$

We remark that there are many systems similar to those we have constructed here with solutions which blow up in finite time. As is clear from the construction, the mechanism for blowup is the reflection of shocks which are stronger than the incident shocks. As in our construction, we can regard such systems as interpolating between $2 \times 2$ systems, with the $2 \times 2$ fluxes chosen so that the interactions have the correct structure. That said, there are clearly some restrictions on the systems that will yield such blowup: indeed, if the system has a uniformly convex entropy, then the $L^{2}$ norm of solutions is bounded, which rules out the blowup we have described here. It is also apparent that a system with a physical meaning, such as the $p$-system of isentropic gas dynamics, cannot be easily adapted to this framework. Indeed, one

${ }^{*}$ I am grateful to James Glimm for pointing out a gap in my original construction here. 
of the components of such systems is usually a velocity, which is generally greater (in absolute value) behind a shock, and can be thought of as driving the shock. In the case of shocks repeatedly being reflected forwards and backwards, the result of a single interaction with a (fixed) standing wave would be a complete reversal of direction of the fluid velocity, which would appear to be impossible on physical grounds.

\section{Instability due to Boundary Effects}

We adapt our example by generating the wave interaction effect at the edge of the strip with a boundary effect, which can be taken to be linear. In doing so, we no longer need the third equation for the variable $S$. Thus, we consider a pair of uncoupled nonlinear Burgers equations,

$$
\begin{aligned}
u_{t}+\left(u^{2} / 2\right)_{x} & =0 \quad \text { and } \\
w_{t}-\left(w^{2} / 2\right)_{x} & =0
\end{aligned}
$$

and for convenience, we restrict to the invariant region

$$
\mathcal{U}=\{(u, w) \mid u \geq 0, w \geq 0\},
$$

which ensures that we have one positive and one negative wavespeed, and fixed boundaries are never characteristic. In particular, we require one boundary condition at each boundary. We will consider both the one-sided boundary condition,

$$
u-\beta w=0 \quad \text { at } \quad x=0,
$$

where $\beta>0$ and the PDE holds on the domain $\Omega_{1}=\{(x, t) \mid x>0, t>0\}$, and the two-sided conditions

$$
\begin{array}{lll}
u-\beta w=0 & \text { at } \quad x=0, & \text { and } \\
w-\beta u=0 & \text { at } \quad x=1,
\end{array}
$$

where the domain of the PDE is the strip $\Omega_{2}=\{(x, t) \mid 0<x<1, t>0\}$.

For this very simple system consisting of a pair of Burgers equations coupled only through linear boundary conditions, our previous construction applies essentially unchanged to (3.1), (3.4) inside the domain $\Omega_{2}$, and we get solutions which blow up in finite time. On the other hand, we will show that the one-sided IBVP is globally wellposed for any initial data. Thus, we draw the important conclusion that for nonlinear problems, it is not enough to consider 'local' boundary conditions when working on a finite domain, and in order to ensure stability, an extra global condition is necessary. Such a condition should be analogous to the existence of an entropy for the Cauchy problem in hyperbolic systems, which implies that the global $L^{2}$-norm of the solution is stable.

3.1. One-sided IBVP. We begin by solving the IBVP (3.1) in the domain $\Omega_{1}$, with boundary condition (3.3), and initial data

$$
\begin{aligned}
& u(x, 0)=u_{0}(x) \quad \text { and } \\
& w(x, 0)=w_{0}(x) \quad \text { for } \quad x>0,
\end{aligned}
$$

and where $u_{0}$ and $w_{0}$ are any nonnegative BV functions. We will see that the boundary condition is taken on in the strong sense, that is pointwise for a.e. $t>0$. 
Our solutions can be constructed either by by Glimm's scheme $[3,10]$ or a front tracking approximation [2], together with an explicit treatment of the boundary effects [4]. Since we have a pair of uncoupled scalar equations and a linear boundary condition, there are no quadratic terms and the Glimm functional is easily bounded.

In the interior of the domain, we make use of the solution to the Riemann problem with states $\left(u_{\ell}, w_{\ell}\right)$ and $\left(u_{r}, w_{r}\right)$. Since the equations (3.1) are uncoupled, we simply solve the Riemann problems for $u$ and $w$ separately. Recalling that we are assuming $u \geq 0$ and $w \geq 0$, we get a forward (resp. backward) wave across which $u$ (resp. $w$ ) changes, while the other variable is constant across the wave. Each wave is a shock or rarefaction depending on whether the wavespeed (which is simply $u$ or $-w$ ) is greater behind or ahead of the wave, and we measure the strength of each wave by

$$
\left|u_{r}-u_{\ell}\right| \text { or }\left|w_{r}-w_{\ell}\right|,
$$

as appropriate. As is well known [10], two forward or backward waves will interact only if one is a shock, and the resulting wave is simply that scalar wave which joins the two exterior states, so that the wave leaving the interaction satisfies

$$
\left|u_{r}-u_{\ell}\right| \leq\left|u_{r}-u_{m}\right|+\left|u_{m}-u_{\ell}\right|,
$$

with the same relation for $w$. Since the two scalar equations are decoupled, they do not interact, and forward and backward waves simply pass through each other unchanged.

In dealing with the boundary, we first solve the boundary Riemann problem (BRP), consisting of the IBVP (3.1), (3.3) and (3.6), and where the initial data is taken to be identically constant,

$$
u_{0}(x)=u_{I} \quad \text { and } \quad w_{0}(x)=w_{I} .
$$

Recall that we are taking $u$ and $w$ to be in the invariant region (3.2), so that we have one characteristic (for $w$ ) leaving the domain, and one (for $u$ ) entering the domain.

We construct a solution to the BRP, where the boundary condition is taken on in the strong sense. Since $w$ does not change across forward waves, the value of $w$ at the boundary should be identically $w_{I}$. Since the boundary condition (3.3) is taken on in the strong sense, we must also have

$$
u(0, t)=\beta w(0, t)=\beta w_{I} .
$$

We now complete the solution to the BRP simply by filling in the forward $u$-wave with $u=\beta w_{I}$ and $u=u_{I}$ behind and ahead of the wave, respectively. That we can do this follows from the fact that both $\beta w_{I}$ and $u_{I}$ are nonnegative. This describes the unique solution to the BRP, and the strength of the outgoing wave is clearly

$$
\left|u_{I}-\beta w_{I}\right| \text {. }
$$

Our existence result is a natural extension of Glimm's theorem.

THEOREM 2. The one-sided IBVP (3.1), (3.3), (3.6) is well-posed in the following sense: For any functions $u_{0}$ and $w_{0}$ of (locally) bounded variation, there is a globally defined weak solution. The boundary condition is taken on in the strong sense, and an appropriately weighted total variation of the solution is non-increasing in time.

Proof. The proof is a standard construction of the solution by Glimm's random choice method [10,3] or front tracking approximations [2]. In the interior, we have two 
uncoupled Burgers' equations, and the waves interact according to (3.8), separately for $u$ and $w$. Since the equations are scalar, there are no quadratic errors in an interaction, and according to (3.8), the total variation of each component decreases after such an interaction.

It remains to treat interactions of waves with the boundary. Clearly only backward waves (across which $w$ changes) enter the boundary, and we must resolve the resulting BRP. A wave approaching the boundary is fully described by specifying an ahead state $\left(u_{a}, w_{a}\right)$ and a single behind state $w_{b}$, and the interaction will be resolved by specifying the wave which is reflected off the boundary. This is accomplished by specifying the state $u_{b}$ behind the reflected wave (since the ahead state is $u_{a}$ ). First, note that since the boundary condition (3.3) is taken on in the strong sense, we must have both

$$
u_{a}=\beta w_{a} \quad \text { and } \quad u_{b}=\beta w_{b},
$$

representing the boundary conditions before and after the interaction, respectively. The outgoing wave is found by resolving the BRP with data $\left(u_{a}, w_{b}\right)$, and the strengths of the outgoing and incoming waves are related by

$$
\left|u_{a}-u_{b}\right|=\beta\left|w_{b}-w_{a}\right| .
$$

We are now in a position to write down the Glimm functional for our system. In the interior, the total variation of $u$ and $w$ are decreasing, and at the boundary, backward $w$ waves are converted to forward $u$-waves after scaling by $\beta>0$. Also, there may be an initial wave entering the domain due to a mismatch between the initial and boundary data. Combining these, we write the discrete functional for the total variation as

$$
\begin{aligned}
G\left(\left\{u_{i}, w_{i}\right\}\right)= & \left|u_{0}-\beta w_{0}\right| \\
& +\sum\left|u_{i}-u_{i-1}\right|+\beta \sum\left|w_{j}-w_{j-1}\right|,
\end{aligned}
$$

where $\left\{u_{i}, w_{i}\right\}$ are the states in the solution, with $u_{0}$ and $w_{0}$ being the leftmost states, although the last term vanishes for $t>0$. We can similarly write down a continuous Glimm functional, namely

$$
\begin{aligned}
G_{c}(t)= & |u(0+, t)-\beta w(0-, t)| \\
& +\int_{0}^{\infty}\left(\left|u_{x}(x, t)\right|+\beta\left|w_{x}(x, t)\right|\right) d x .
\end{aligned}
$$

It is now standard to show that these functionals are non-increasing and bounded by the initial data, and the conclusions of the theorem follow. Details are left to the reader. $\mathrm{c}$

It is clear that the IBVP is stable for all values of $\beta>0$, and that the same result holds for a linear boundary condition at the right edge of an unbounded domain $x<0$. We also note that we have no restrictions on the size of the initial data, and that uniqueness and continuous dependence follow as for scalar equations [2].

3.2. Two-sided IBVP. Having established well-posedness of the IBVP for a one-sided domain, we now consider the IBVP on a compact domain, subject to the two-sided boundary condition (3.4). We wish to mirror our earlier unstable construction in which the solution blows up in finite time. As in that case, it is most 
convenient to take constant initial data, and choose this in such a way that only one shock enters the domain initially.

We thus choose the initial condition

$$
u_{0}(x)=u_{0}=u_{I}, \quad \text { and } \quad w_{0}(x)=w_{0}=\beta u_{I},
$$

where $u_{I}>0$ is arbitrary. For $\beta>1$, we again get blowup in finite time, and indeed the blowup is faster here than previously.

TheOrem 3. The two-sided IBVP (3.1), (3.4) with $\beta>1$ is ill-posed. For initial data (3.16), the solution blows up in finite time on the whole support of the initial data. In particular, no integral norm is finite, and the solution cannot be extended beyond the time of blowup.

Proof. First we note that (3.16) is consistent with (3.4) at the right boundary $x=1$, so no wave enters the domain from the right-hand corner of the domain. Therefore, there is only a single forward wave in the solution initially, generated by the mismatch of the initial and boundary conditions at the origin. According to our solution (3.10) of the BRP, the state behind this wave is

$$
u_{1}=\beta w_{0}=\beta^{2} u_{0}>u_{0},
$$

so this wave is a shock.

Referring to Fig. 2.1 for notation, we now proceed inductively. Each forward shock meets the right boundary and reflects a backward wave. Thus if the shock has states $\left(u_{i}, w_{i}\right)$ and $\left(u_{i+1}, w_{i}\right)$ ahead of and behind the wave, respectively, then the reflected wave will have $\left(u_{i+1}, w_{i+1}\right)$ behind it, where

$$
w_{i+1}=\beta u_{i+1}=\beta^{2} w_{i}>w_{i},
$$

and so the reflected wave is also a shock. By induction, we thus have

$$
u_{i+1}=\beta w_{i} \quad \text { and } \quad w_{i+1}=\beta u_{i+1},
$$

and so in particular

$$
u_{n}=\beta^{2 n} u_{I} \quad \text { and } \quad w_{n}=\beta^{2 n+1} u_{I} .
$$

The rest of the proof now proceeds as before, with equations (2.33), (2.34) and (2.35) holding identically, while (2.36) becomes

$$
\begin{aligned}
t_{2 J} & =\frac{2}{u_{0}+u_{1}} \sum_{j \leq J} \beta^{-2 j}+\frac{2}{w_{0}+w_{1}} \sum_{j \leq J} \beta^{-2 j} \\
& \leq \frac{2}{u_{I}} \frac{1}{1+\beta} \sum_{k} \beta^{-k} \leq \frac{2}{u_{I}} \frac{\beta}{\beta^{2}-1}
\end{aligned}
$$

and the conclusions of the theorem follow.

\section{Blowup of Compressive Waves}

We have seen that genuine nonlinearity is an essential ingredient of our construction, in that the increasing speeds of reflected shocks leads to infinitely interactions in finite time. Here we show that the presence of shocks and the piecewise constant character of the solution are not necessary for blowup. We study a solution consisting 
of compressive waves, and show that it blows up before the compressive waves break down and form shocks. Clearly the gradients must increase, but they only become infinite when the solution itself does. This shows that the cause of instability is the nonlinear coupling of different families together with genuine nonlinearity, and blowup of amplitude is a separate phenomenon from shock formation. For an analysis of other mechanisms of blowup, including 'gradient driven blowup', in which the steepening of gradients and shock formation drives an infinite growth in amplitude, see [7].

Our solution is not smooth in the Riemann coordinate $S$, since we need jump discontinuities to effect the immediate change of flux at the ends of the strip $0<x<1$. If $S$ changes smoothly but rapidly, resonances could develop inside the standing wave, resulting in a much more complicated wave pattern. Moreover, by using compressive waves, we would normally get an interaction zone in which forward and backward waves interact nonlinearly, and in which it would be much more difficult to resolve the solution exactly. Here, we have $S=1$ inside the strip, so the reduced $2 \times 2$ system is uncoupled, and we can treat the compressive waves exactly. We expect that for reasons of structural stability, solutions should still blow up if the jump discontinuities were smoothed out. It is an interesting and challenging computational problem to study these interactions and find blowup numerically.

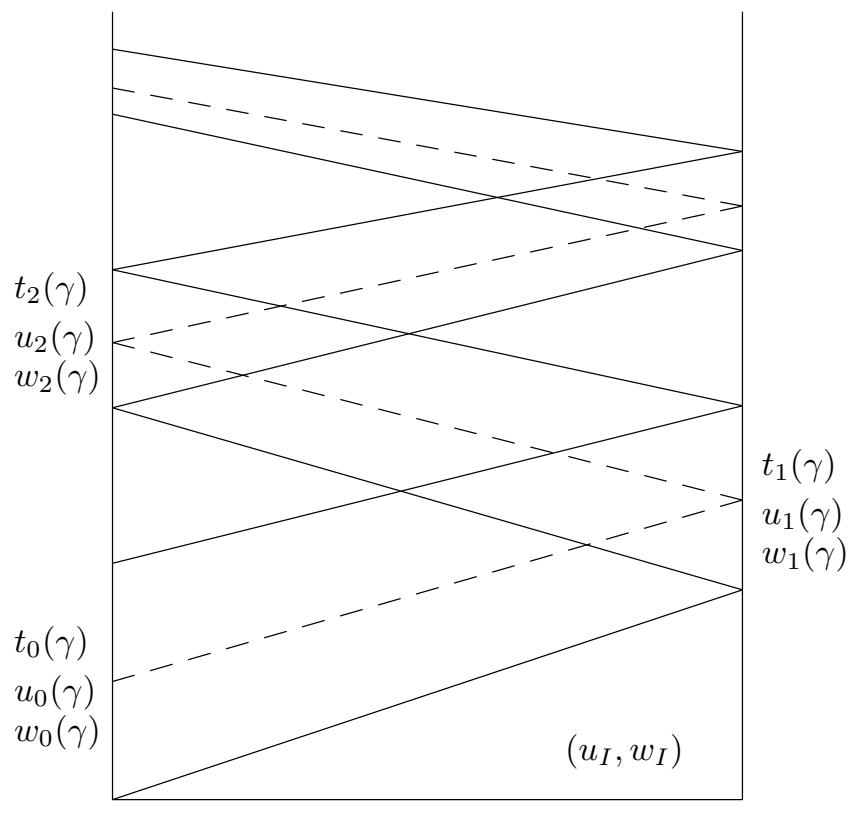

FIG. 4.1. The Compressive Case

Referring to Fig. 4.1, we concentrate on the solution inside the strip, consisting of multiply reflected compressive waves. We parameterize the compression wave at $x=0$ or $x=1$ by $t=t_{i}(\gamma)$ and $\left(u_{i}(\gamma), w_{i}(\gamma)\right)$ for $\gamma \in[0,1]$, where the subscript denotes the number of reflections the compression wave has gone through. The states are the limits from the inside of the strip, and we use the same parameter $\gamma$ throughout by following it along forward or backward characteristics. Thus, the states satisfy

$$
u_{2 k+1}(\gamma)=u_{2 k}(\gamma) \text { and } w_{2 k+2}(\gamma)=w_{2 k+1}(\gamma),
$$


while the times at which the characteristics intersect with the edges satisfy

$$
\begin{aligned}
& t_{2 k+1}(\gamma)=t_{2 k}(\gamma)+\frac{1}{u_{2 k}(\gamma)} \text { and } \\
& t_{2 k+2}(\gamma)=t_{2 k+1}(\gamma)+\frac{1}{w_{2 k+1}(\gamma)}
\end{aligned}
$$

since the characteristic speeds are $u$ and $-w$, respectively.

We now use the jump conditions (2.10) at the edges of the strip to relate $u_{i}(\gamma)$ to $w_{i}(\gamma)$. Following our previous argument, we again get (2.16), where now the states are varying and parameterized by $\gamma$. In our current notation, we rewrite this as

$$
\left(\begin{array}{c}
\frac{1}{2} u_{i}^{2}(\gamma) \\
-\frac{1}{2} w_{i}^{2}(\gamma)
\end{array}\right)=A\left(\begin{array}{c}
\bar{u}_{i}(\gamma) \\
\bar{w}_{i}(\gamma)
\end{array}\right)
$$

where the bar refers to the corresponding limits on the outside edge of the strip. Again as before, we now use the fact that the wave leaving the strip is a forward linear wave on the right (when $i$ is odd), or a backward wave. Since the flux is linear outside the strip, this means that the change in state vector $(\bar{u}, \bar{w})$ is parallel to the eigenvector $r_{+}$(resp. $r_{-}$) on the right (resp. left) of the strip,

$$
\frac{d}{d \gamma}\left(\begin{array}{c}
\bar{u} \\
\bar{w}
\end{array}\right)=\alpha r_{ \pm}
$$

or equivalently

$$
\ell_{-} \cdot \frac{d}{d \gamma}\left(\begin{array}{c}
\bar{u}_{2 k+1} \\
\bar{w}_{2 k+1}
\end{array}\right)=0 \quad \text { and } \quad \ell_{+} \cdot \frac{d}{d \gamma}\left(\begin{array}{c}
\bar{u}_{2 k+2} \\
\bar{w}_{2 k+2}
\end{array}\right)=0
$$

for the forward and backward linear waves, respectively. Now using the jump conditions (4.3) to relate the waves outside the strip to the interior waves, we get

$$
\ell_{-} \cdot \frac{d}{d \gamma}\left(\begin{array}{c}
u_{2 k+1}^{2} \\
-w_{2 k+1}^{2}
\end{array}\right)=0 \quad \text { and } \quad \ell_{+} \cdot \frac{d}{d \gamma}\left(\begin{array}{c}
u_{2 k+2}^{2} \\
-w_{2 k+2}^{2}
\end{array}\right)=0
$$

or equivalently by $(2.23)$,

$$
\begin{aligned}
& \frac{d}{d \gamma}\left(w_{2 k+1}^{2}-\beta u_{2 k+1}^{2}\right)=0, \quad \text { and } \\
& \frac{d}{d \gamma}\left(u_{2 k+2}^{2}-\beta w_{2 k+2}^{2}\right)=0,
\end{aligned}
$$

at the right and left inside edges of the strip, respectively.

Integrating (4.7), we get the relations

$$
\begin{aligned}
& w_{2 k+1}^{2}(\gamma)-\beta u_{2 k+1}^{2}(\gamma)=c_{2 k+1} \quad \text { and } \\
& u_{2 k+2}^{2}(\gamma)-\beta w_{2 k+2}^{2}(\gamma)=c_{2 k+2},
\end{aligned}
$$

respectively, for some constants $c_{i}$. We now impose the condition that there are no waves other than those we have identified: these are the conditions

$$
u_{2 k+2}(0)=u_{2 k}(1) \quad \text { and } \quad w_{2 k+1}(0)=w_{2 k-1}(1),
$$


which simply say that the state behind one forward or backward wave is the state ahead of the next such wave. Using (4.9) and (4.1) in (4.8), we get

$$
\begin{aligned}
c_{2 k+2} & =u_{2 k+2}^{2}(0)-\beta w_{2 k+2}^{2}(0) \\
& =u_{2 k}^{2}(1)-\beta w_{2 k}^{2}(1) \\
& =c_{2 k},
\end{aligned}
$$

and similarly $c_{2 k+1}=c_{2 k-1}$ for each $k$. Thus, the constants in (4.8) are either $c_{0}$ or $c_{1}$, and we can write

$$
c_{i}=\frac{c_{0}+c_{1}}{2}+(-1)^{i} \frac{c_{0}-c_{1}}{2}
$$

for each $i$.

We now define

$$
z_{i}(\gamma)= \begin{cases}u_{i}^{2}(\gamma), & \text { for } i \text { even } \\ w_{i}^{2}(\gamma), & \text { for } i \text { odd }\end{cases}
$$

and use (4.1) to rewrite (4.8) as

$$
z_{i+1}(\gamma)=\beta z_{i}(\gamma)+c_{i+1},
$$

for both even and odd values of $i$. We solve this by writing

$$
z_{i+1}+a+(-1)^{i+1} b=\beta\left(z_{i}+a+(-1)^{i} b\right),
$$

which immediately yields

$$
z_{n}(\gamma)+a+(-1)^{n} b=\beta^{n}\left(z_{0}(\gamma)+a+b\right) .
$$

Here, using (4.11), the constants $a$ and $b$ are given by

$$
\begin{aligned}
c_{i+1} & =a(\beta-1)+(-1)^{i} b(\beta+1) \\
& =\frac{c_{0}+c_{1}}{2}+(-1)^{i+1} \frac{c_{0}-c_{1}}{2},
\end{aligned}
$$

these being determined by our initial parameterization of the compressive wave.

Having solved (4.13), we rewrite (4.2) as

$$
t_{i+1}(\gamma)=t_{i}(\gamma)+\frac{1}{\sqrt{z_{i}(\gamma)}}
$$

which we immediately solve to get

$$
t_{n}(\gamma)=t_{0}(\gamma)+\sum_{i<n} \frac{1}{\sqrt{z_{i}(\gamma)}}
$$

for all $n$. Our solution (4.15) implies that $z_{n} \rightarrow \infty$ as $n \rightarrow \infty$ for $\beta>1$, so the solution blows up, and this occurs in finite time if the sum (4.18) converges. Moreover

$$
\frac{1 / \sqrt{z_{n+1}}}{1 / \sqrt{z_{n}}} \rightarrow \frac{1}{\sqrt{\beta}}<1
$$


so convergence of the sum (4.18) follows from the ratio test, and the blowup does indeed take place in finite time.

It remains to show that we can consistently choose our data so that this blowup takes place before the compression collapses and a shock is formed. We need to check two conditions, namely that successive forward or backward waves do not overlap, or equivalently

$$
t_{i+2}(0) \geq t_{i}(1)
$$

for each $i$, and secondly that the characteristics inside each wave do not overlap, which is the condition

$$
\frac{d t_{i+1}}{d \gamma} \geq 0 \quad \text { for every } \quad i \geq 0 .
$$

Together these imply that the blowup time $t_{*}$ is uniquely defined, that is

$$
t_{n}(\gamma) \rightarrow t_{*} \quad \text { for each } \gamma \in[0,1]
$$

with $t_{*}$ independent of $\gamma$. Taking the limit of (4.18), we thus see that $t_{0}(\gamma)$ is defined by

$$
t_{0}(\gamma)=t_{*}-\sum_{i \geq 0} \frac{1}{\sqrt{z_{i}(\gamma)}}
$$

and using this in (4.18), we have

$$
t_{n}(\gamma)=t_{*}-\sum_{i \geq n} \frac{1}{\sqrt{z_{i}(\gamma)}}
$$

for each $n$. Differentiating (4.24), we get

$$
\frac{d}{d \gamma} t_{n}(\gamma)=\frac{1}{2} \sum_{i \geq n} \frac{z_{i}^{\prime}(\gamma)}{{\sqrt{z_{i}(\gamma)}}^{3}}=\frac{z_{0}^{\prime}(\gamma)}{2} \sum_{i \geq n} \frac{\beta^{i}}{{\sqrt{z_{i}(\gamma)}}^{3}}
$$

where we have used (4.15), so that (4.21) holds for each $n$. On the other hand, using (4.24) in (4.20) gives

$$
t_{n+2}(0)-t_{n}(1)=\sum_{i \geq n} \frac{1}{\sqrt{z_{i}(1)}}-\sum_{i \geq n+2} \frac{1}{\sqrt{z_{i}(0)}}=0
$$

where we have used (4.9), which we write as $z_{j+2}(0)=z_{j}(1)$. Thus, (4.21) is satisfied as an equality, and there is no strict separation of successive waves.

We summarize the steps in setting up this construction. Our goal is to choose the profile of the initial compression wave in such a way that the blowup of derivatives and amplitude is simultaneous. Start by choosing a positive increasing function $h(\gamma)$,

$$
h(\gamma)>0, \frac{d h}{d \gamma}>0 \quad \text { for } \quad 0 \leq \gamma \leq 1
$$

We will set $z_{0}(\gamma)=h(\gamma)$, and using (4.15),

$$
z_{n}(\gamma)=\beta^{n}\left(h(\gamma)+h_{+}\right)-h_{(-)^{n}}
$$


where $h_{ \pm}=a \pm b$ are constants. Requiring $z_{2}(0)=z_{0}(1)$ leads to

$$
h(1)=\beta^{2}\left(h(0)+h_{+}\right)-h_{+}, \quad \text { so that } \quad h_{+}=\frac{h(1)-\beta^{2} h(0)}{\beta^{2}-1},
$$

while the requirement $z_{1}(0)>0$ becomes

$$
0<\beta\left(h(0)+h_{+}\right)-h_{-}, \quad \text { so that } \quad h_{-}<\frac{\beta}{\beta^{2}-1}(h(1)-h(0)),
$$

where we have used (4.29). Thus, the constant $h_{-}$can be arbitrarily chosen subject to $(4.30)$. Now the time of blowup $t_{*}$ is given by

$$
t_{*}=\sum_{i} \frac{1}{\sqrt{z_{i}(0)}}
$$

and the parameterization $\tau(\gamma) \equiv t_{0}(0)$ is given by (4.23),

$$
\tau(\gamma)=t_{*}-\sum_{i} \frac{1}{\sqrt{z_{i}(\gamma)}}
$$

This completes our specification of the initial compressive wave on the left of the strip, $x=0$. It is clear that by tracing characteristics forward or backward in time, we can prescribe Cauchy data for an initial value problem.

Note that we still have the freedom to choose the initial parameterization $h(\gamma)$. Given (constant) initial states $u_{0}, u_{1}>u_{0}$ and $w_{0}$, and referring to our definition (4.12), if we choose

$$
h(0)=u_{0}^{2} \quad \text { and } \quad h(1)=u_{1}^{2},
$$

then $\gamma$ parameterizes the compression with ahead state $u_{0}$ and $u_{1}$ behind. According to $(4.29)$, we get

$$
h_{+}=\frac{u_{1}^{2}-\beta^{2} u_{0}^{2}}{\beta^{2}-1},
$$

and to ensure that $z_{1}(0)=w_{0}^{2}$ in $(4.30)$, we set

$$
h_{-}=\frac{\beta}{\beta^{2}-1}\left(u_{1}^{2}-u_{0}^{2}\right)-w_{0}^{2} .
$$

Using (4.33), (4.34) and (4.35) in (4.28) and (4.32), we can give $\tau=\tau(u)$ as the parameterization of the compression wave.

In particular, if we set

$$
u_{1}=\beta u_{0} \quad \text { and } \quad w_{0}=\sqrt{\beta} u_{0}
$$

then we get $h_{+}=h_{-}=0$, and our description simplifies. It is convenient to set

$$
h(\gamma)=\beta^{\gamma} u_{0}^{2},
$$

so that

$$
z_{n}(\gamma)=\beta^{n+\gamma} u_{0}^{2}
$$


This in turn leads to

$$
t_{*}=\frac{1}{u_{0}} \sum_{i} \frac{1}{\sqrt{\beta}^{i}}=\frac{1}{u_{0}} \frac{\sqrt{\beta}}{\sqrt{\beta}-1}
$$

and similarly by (4.24),

$$
\tau_{n}(\gamma)=t_{*}\left(1-\frac{1}{\sqrt{\beta}^{n+\gamma}}\right)=t_{*}\left(1-\frac{u_{0}}{\sqrt{z_{n}(\gamma)}}\right) .
$$

We can now express the solution explicitly everywhere inside the strip: noting that $z_{i}=u_{i}^{2}$ for $i$ even, we simply set

$$
t(u)=t_{*}\left(1-\frac{u_{0}}{u}\right) \quad \text { or } \quad u(t)=\frac{u_{0} t_{*}}{t_{*}-t}
$$

where here $u=u(t)=u(0, t)$ is the value of $u$ at the left edge, which propagates along the forward characteristic having slope $1 / u$, which corresponds to speed $u$. The characteristic through the point $(0, \bar{t})$ can thus be written

$$
t=\bar{t}+\frac{x}{u(\bar{t})}=\bar{t}+\frac{x\left(t_{*}-\bar{t}\right)}{u_{0} t_{*}}, \quad \text { or } \quad x=u_{0} t_{*} \frac{t-\bar{t}}{t_{*}-\bar{t}}
$$

and on this characteristic we have

$$
u(x, t)=u(\bar{t})=\frac{u_{0} t_{*}}{t_{*}-\bar{t}} .
$$

It is easy to calculate the point of intersection of any two of these characteristics, or simply observe from (4.42) that all of them pass through the point

$$
t=t_{*}, x=u_{0} t_{*}=\frac{\sqrt{\beta}}{\sqrt{\beta}-1}>1,
$$

which in particular is outside the strip $0 \leq x \leq 1$. We conclude that inside the strip, the forward compression wave is that part of the infinite Burgers' compression wave which is centered at the point $\left(\frac{\sqrt{\beta}}{\sqrt{\beta}-1}, t_{*}\right)$.

We can similarly calculate the backward compression wave, but it is easier to use symmetry. That is, our backward compression wave for $w$ is the trace of the infinite backward compression wave centered at the point $\left(1-\frac{\sqrt{\beta}}{\sqrt{\beta}-1}, t_{*}\right)$. Thus, the characteristics are the lines through this point, and corresponding value of $w$ is the absolute speed of the characteristic. Thus, each characteristic is given by

$$
x+\frac{1}{\sqrt{\beta}-1}=-w\left(t-t_{*}\right)
$$

and so the solution is

$$
w(x, t)=\left(x+\frac{1}{\sqrt{\beta}-1}\right) \frac{1}{t_{*}-t} .
$$

As a final check, we verify that our solution inside the strip satisfies the correct jump condition at the right edge $x=1$. To do so, we fix some $\tilde{t}$ and calculate $\tilde{u}=u(1, \tilde{t})$ and $\tilde{w}=w(1, \tilde{t})$. From (4.46), we get

$$
\tilde{w}=\frac{\sqrt{\beta}}{\sqrt{\beta}-1} \frac{1}{t_{*}-\tilde{t}}
$$


and since also

$$
1-\frac{\sqrt{\beta}}{\sqrt{\beta}-1}=\tilde{u}\left(\tilde{t}-t_{*}\right)
$$

we get

$$
\tilde{u}=\frac{1}{\sqrt{\beta}-1} \frac{1}{t_{*}-\tilde{t}}=\frac{\tilde{w}}{\sqrt{\beta}},
$$

so the solution satisfies the jump condition (4.4) as expected.

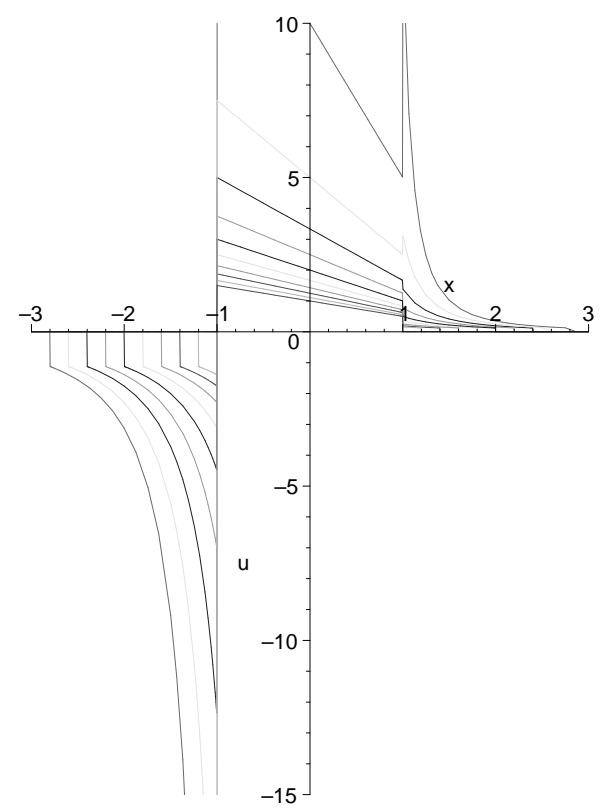

FIG. 4.2. Snapshots of the solution $u(x, t)$

Figure 4.2 shows the profile of the first component $u$ of our solution at various positive times. It is apparent that most of the growth of $u$ is driven by interactions of $w$-waves with the jump in $S$ at $x=-\ell$; for fixed $x$, it is also clear that the solution is monotone in time. The vertical lines are plotting anomalies.

To find Cauchy data which leads to blowup, we simply take the trace of our compressions on the Cauchy curve inside the strip, and it is enough to take the data to be constant outside the strip as in the previous case. We find the linear waves which leave the strip in the same way as before, by solving equations (4.3) at each edge. We omit the details.

THEOREM 4. There are Cauchy data for equation (2.8) which are continuous in the genuinely nonlinear fields, and for which finite time blowup in amplitude occurs without prior formation of shocks. In fact, the solution is smooth away from the jumps in $S$ at $x=0$ and $x=1$. The same comments hold for solutions of the IBVP (3.1), (3.4).

The only change in the above construction necessary for the IBVP is to replace $\sqrt{\beta}$ by $\beta$ as before. Our construction does suggest that repeated interactions with jump 
discontinuities could sustain and strengthen a compression wave until blowup occurs in a number of situations. In particular, the blowup is stable under perturbations of $u$ and $w$ subject to (4.27) and (4.30). However, we again note that smoothing out the jump discontinuity may lead to some internal resonances which certainly complicate the wave pattern, and may slow down blowup. Indeed, if our solution were mollified, then there must be some point in the solution at which strict hyperbolicity fails, as each of $u$ and $w$ do change sign at one of the edges of the strip (although the system remains strongly hyperbolic). This loss of strict hyperbolicity will be studied further in $[12]$.

\section{REFERENCES}

[1] P. Baiti, and H.K. Jenssen, Blowup in $L^{\infty}$ for a class of genuinely nonlinear hyperbolic systems of conservation laws. Discrete and Continuous Dynamical Systems Ser, 7(A):837-853, 2001.

[2] A. Bressan, Hyperbolic Systems of Conservation Laws: The One-Dimensional Cauchy Problem. Oxford University Press, 2000.

[3] J. Glimm, Solutions in the large for nonlinear hyperbolic systems of equations. Comm. Pure Appl. Math., 18:697-715, 1965.

[4] J.B. Goodman, Initial Boundary Value Problems for Hyperbolic Systems of Conservation Laws. Ph.D. thesis, Stanford University, 1982.

[5] J. Hunter, Strongly nonlinear hyperbolic waves. Nonlinear Hyperbolic Equations - Theory, Computation Methods, and Applications (J. Ballmann \& R. Jeltsch, ed.), Viewig, 257268,1989

[6] H.K. Jenssen, Blowup for systems of conservation laws. SIAM J. Math. Anal., 4(31):894-908, 2000.

[7] H.K. Jenssen and R.C. Young, Mechanisms for blowup in conservation laws. in preparation, 2002.

[8] J.L. Joly, G. Metivier, and J. Rauch, A nonlinear instability for $3 \times 3$ systems of conservation laws. Commun. Math. Physics., 162:47-59, 1994.

[9] P.D. Lax, Hyperbolic systems of conservation laws, II. Comm. Pure Appl. Math., 10:537-566, 1957.

[10] J. Smoller, Shock Waves and Reaction-Diffusion Equations. Springer-Verlag, New York, 1982.

[11] Bo Su, Stability of solutions to Hamilton-Jacobi equations. Proceedings of the Ninth International Conference on Hyperbolic Problems, Springer, Submitted.

[12] R. Young and W. Szeliga, Blowup with small BV data in hyperbolic conservation laws. submitted.

[13] R. Young, On elementary interactions for hyperbolic conservation laws. Preprint, 1993.

[14] _ Sup-norm stability for Glimm's scheme. Comm. Pure Appl., Math., 46:903-948, 1993.

[15] _ Exact solutions to degenerate conservation laws. SIAM J. Math. Anal., 30:537-558, 1999. 